\section{Minimum-Coulomb-energy electrostatic configurations}

SIR-Determination of the stable (least energy) configuration of $N$ equal charges on the surface of a unit sphere remains incomplete ${ }^{1}$ despite the importance of the problem in botany, nuclear theory and elsewhere ${ }^{1,2}$. Recently Munera ${ }^{2}$ presented an elegant description of three-dimensional configurations in which the charges are partitioned among the vertices of regular polygons, parallel to the equatorial plane of the sphere. Munera labels the results as possessing minimum Coulomb energy. But configurations with lower energies can be found. Munera's Table 2 should therefore be more clearly labelled as the minimum energies obtained by numerical optimization subject to the strict (and over-limiting) geometrical constraints applied. Indeed Munera seems to acknowledge the existence of a lower energy distribution for $N=11$ and also states that "rotational equilibrium does not imply minimum energy". Here it is indeed true that the solutions presented improve on several previously reported, and conveniently involve vertices of polyhedra with triangular faces, but they are nevertheless suboptimal and derivative physical interpretations are inaccurate.

Truly optimal configurations have been obtained by numerical Monte Carlo techniques in which no constraints on geometric regularity are applied. Whilst one must accept that the results of such a technique only generate true minima for infinite computing time, one cannot dispute that the configurations generated by finite computations are more stable and lower energy configurations than those calculated by Munera have been found for $N=11,13$, $15,19,20$. As an example a configuration with $W(13)=58.8535 \quad$ (origin = sphere centre) is given below:

\begin{tabular}{ccc}
$x$ & $y$ & $z$ \\
0.00000 & 0.00000 & 1.00000 \\
-0.22004 & -0.97369 & -0.05925 \\
-0.79907 & -0.31927 & 0.50946 \\
0.78227 & 0.38408 & 0.49045 \\
0.21185 & -0.51330 & -0.83165 \\
-0.20457 & 0.40678 & -0.89033 \\
0.87263 & -0.48031 & -0.08844 \\
-0.30184 & 0.73503 & 0.60715 \\
0.75763 & 0.33346 & -0.56107 \\
0.18079 & 0.97225 & -0.14849 \\
0.34187 & -0.71106 & 0.61443 \\
-0.77193 & -0.35116 & -0.52992 \\
-0.84741 & 0.51760 & -0.11829 \\
\hline
\end{tabular}

Removal of the symmetry constraint denies a simple geometrical shorthand but the result cannot be ignored.

Munera's demonstration that the effective equipotential surface resulting from a distribution of finite charges can lead to classical transparency to a non-relativistic particle is exciting in view of a novel way of interpreting nuclear scattering and radioactive decay. The prediction of a finite number of directions in which 'holes' in the equipotential surface arise may, however, be a due to the assumed symmetries and before further physical interpretations it is important to establish whether the same phenomena arise when the constraint is removed as for example with the numerical data reported here.

Joint Section of Physics, $S$ WEBB

Institute of Cancer Research and Royal Marsden Hospital, Sutton, Surrey SM2 5PT, UK

1. Berezin, A.A. Chem. Phys. Lett. 123, (1,2), 62-64 (1986) 2. Munera, H.A. Nature 320,597-600 (1986)

\section{Haldane's rule back in the news}

SIR-There has been a revival of interest in Haldane's rule ${ }^{1,2}$; that "When in the F offspring of two different animal races one sex is absent, rare or sterile, that sex is the heterozygous (heterogametic) sex" 3 . Haldane felt that if his rule "is more than a mere coincidence" it must show how lethality, sterility and, in some instances, the sex transformation of the heterogametic sex in hybrids, "may be explained as due to the same cause" ${ }^{3}$. In trying to understand the genetic basis of Haldane's rule, it might be illuminating to view such sex-specific fitness effects (in the offspring of interspecific hybrids) in the larger context of sex-specific differences related to mechanisms of sex determination.

Haldane preferred not to accept the obvious explanation in terms of sex factors because clear-cut examples of such genes were unknown. He sought an explanation in terms of autosomal genes, a few of which are known to cause sex-specific effects on fitness. Properties of genes such as daughterless (da) and male-lethal (mle) in Drosophila melanogaster take on added significance in the light of Haldane's views. These genes are autosomal but they are part of the sex-determination mechanism and appear to act to cause sexspecific lethality ${ }^{46}$.

Haldane maintained that heterogameity rather than sex is the critical feature responsible for the sex-specific fitness effects observed in interspecific hybrids ${ }^{3}$. It may therefore be of interest to note that according to a model developed by us to understand $\mathrm{X} / \mathrm{A}$ ratio measurement and the effects of $d a$, mle and certain mutations at the X-linked locus sex-lethal, the narrow range of viability of the male $D$. melanogaster is a consequence of his heterogametic condition ${ }^{6}$.

It appears that sex transformation of the heterogametic hybrid - more than its lethality or sterility - requires explanation in terms of incompatibility between the sex-determining mechanisms of the parental species or races. A case of sex reversal in hybrid mice ${ }^{7}$ has been inter- preted on this basis ${ }^{8}$. Data from these hybrid mice, in particular differences between the $F_{1}$ and backcross progeny in the frequency and degree of sex transformation, may be explained as being due to genetic interaction among an autosomal gene, an X-linked gene and the Y chromosome $e^{8}$

$M C B L \& I C M R$ Centre,

H. Sharat Chandra

Indian Institute of Science,

Bangalore 560012 ,

India

1. Coyne, J.A. Nature 314, 736-738 (1985)

2. Licht, L.E. \& Bogart. J.P. Nature 317, 207 (1985).

3. Haldane, J.B.S. J. Genet. 12, 101-109 (1922)

4. Cline, T.W. Genetics $90,683-698(1978)$

Belote, J.A. \& Lucchesi, J.C. Nature 285, 573.575 (1980).

6. Gadagkar, R., Nanjundiah, V., Joshi, N.V. \& Chandra, H.S. J. Biosci. 4, 393-415 (1982); Curr. Sci. 50, 805-807 (1981)

. Eicher, E.M., Washburn, L.L., Whitney, J.B. \& Morrow, K.E. Science 217, 535-537 (1982)

8. Chandra, H.S. Molec. gen. Genet. 192, 384-388 (1984).

\section{Radiation and fetal brain development}

Sir-Your article about the reactor accident at Chernobyl' ignores the fact that radiation sickness, cancer and mutations are not the only important effects of excessive radioactivity.

Recent studies in our laboratory ${ }^{2-4}$ and reports from others have revealed that radiation exposure of pregnant animals produces permanent brain deficiencies in their offspring, even at doses too low to produce radiation sickness. Early exposures are particularly damaging, because radiation affects DNA replication in the early proliferation of neurones. Since, in humans, neurone proliferation terminates before the eighteenth week of pregnancy radiation exposure may be particularly harmful at a time when a woman may not yet know that she is pregnant. Thus an evacuation of women known to be pregnant (as happened at Chernobyl), even if it can be accomplished immediately, would still leave those women unaware of their pregnancy at risk of fetal brain damage.

It is important to note that, in contrast to cancer and genetic damage (mutations), which are rare events, brain damage could occur in almost every fetus exposed to radiation. Mental Retardation Research Center and Brain Research Institute.

UCLA School of Medicine,

Los Angeles, California 90024,

USA

1. Nature 321, 100 (1986)

2. Zamenhof, S. \& van Marthens, E. Radiat. Res. 77, 117-127 (1979)

3. Zamenhof, S. \& van Marthens, E. Radiat. Res. 85, 292-301 (1981).

4. van Marthens, E. \& Zamenhof, S. in Developmental Effects of Prenatal Irradiation (eds Kriegel, H., Schmal, W. Kistner, G. \& Stieve, F.E.) 92-103 (Gustav Fisher, New York, 1982). 